\title{
OSZE-Friedensmissionen: Bilanz und Zukunftsperspektiven
}

\author{
Fred Tanner"
}

\section{Zusammenfassung}

Die Sonderbeobachtermission in der Ukraine (SMM) ist eine der wenigen Erfolgsgeschichten der OSZE seit dem Beginn des bewaffneten Konfliktes in der Ostukraine. Die zivile Mission war und ist auf einzigartige Weise in der Lage, in der Hochrisikoregion des Donbass als Quasi-Friedenstruppe tätig zu sein. Sie beobachtet die Einhaltung von Waffenstillständen, fördert Dialog und bietet humanitäre Hilfeleistungen für die in der Konfliktzone lebenden Menschen. In der Geschichte der OSZE gab es zuvor nur eine weitere Mission zur Beobachtung von Waffenstillständen, die Kosovo-Verifikationsmission (KVM). Der vorliegende Beitrag zieht - unter besonderer Berücksichtigung dieser beiden Missionen - Bilanz aus den OSZE-Friedensoperationen. Alle bisherigen OSZE-Friedensoperationen waren zivile Missionen, und militärische OSZE-Missionen sind auch in Zukunft schwer vorstellbar. Dieser Beitrag präsentiert Erfahrungen und Empfehlungen zur Verbesserung von zukünftigen Missionen der OSZE und von anderen Organisationen, die in einem Hochrisiko-Umfeld tätig sind.

\section{Schlagworte}

OSZE, Friedensoperationen, Kosovo Verifikationsmission, Sonderbeobachtermission in der Ukraine

Bitte zitieren als: Fred Tanner, OSZE-Friedensmissionen: Bilanz und Zukunftsperspektiven, OSCE Insights 4/2021 (Baden-Baden: Nomos, 2021), https://doi.org/10.5771/9783748911449-04

\section{Einleitung}

Welche Erfahrungen hat die OSZE bei zivilen Friedensoperationen in HochrisikoGebieten gewonnen? ${ }^{1}$ Solche Missionen sind ein wesentlicher Bestandteil der internationalen Bemühungen um Konfliktverhütung, Krisenmanagement und Frie-

\section{* Fred Tanner}

The Graduate Institute, Centre on Conflict, Development \& Peacebuilding, Genf fred.tanner@graduateinstitute.ch densaufbau. Laut des Stockholm International Peace Research Institutes wurden im Jahr 2020 weltweit 62 Friedensmissionen entsandt - 25 davon unter Leitung der Vereinten Nationen und 16 unter Leitung der OSZE. ${ }^{2}$ Auf den ersten Blick scheint es, als habe eine multidimensionale UN-Friedensmission in Somalia wenig mit einer OSZE-Operation auf dem Westbalkan oder in Zentralasien gemein. Allerdings würden die meisten OSZE-Feldmissionen durchaus als das durchgehen, was die Hochrangige Unabhängige Gruppe für Friedensmissionen 
der Vereinten Nationen als „Friedensoperation" bezeichnet - auch wenn die Friedensoperationen der OSZE keine „robusten" Elemente wie etwa den Schutz von Zivilisten enthalten. Es ist nach wie vor unwahrscheinlich, dass es jemals eine OSZE-Mission mit Beteiligung von Polizei- oder bewaffneten Armee-Einheiten geben wird. Allerdings wird diese Möglichkeit seit der Genehmigung von Feldmissionen durch das Dokument von Helsinki von 1992 diskutiert. ${ }^{3}$

Die Möglichkeit einer militärischen Friedensmission durch die OSZE ist nur ein einziges Mal erwogen worden, und zwar von einer Hochrangigen Planungsgruppe (HLPG, High-Level Planning Group) im Rahmen der 1995 gegründeten und von der OSZE geleiteten Minsker Vermittlungsplattform zum Bergkarabach-Konflikt. In Erwartung eines Friedensvertrags zwischen Armenien und Aserbaidschan entwickelte die HLPG vier verschiedene Optionen für eine solche Mission. Die robusteste davon beinhaltete die Entsendung bewaffneter, aus Infanteriebataillonen bestehender Kontingente, die fast 5000 Soldaten umfasst hätten. Die Bemühungen um eine Friedensmission der OSZE nahmen Ende 2020 jedoch ein plötzliches Ende, als Aserbaidschan sein Territorium gewaltsam zurückeroberte und Russland ein nationales Kontingent zur Friedenssicherung in die Region schickte.

\section{Die Erfahrung der OSZE mit Friedensmissionen}

Welche politischen und operativen Erkenntnisse konnten im Rahmen der OSZE-Missionen gewonnen werden? Und wie können diese Erkenntnisse die Effektivität sowie die politische und operative Durchführbarkeit zukünftiger Friedensmissionen verbessern?

In den letzten 25 Jahren enthielten die Mandate mehrerer Feldoperationen polizeiliche oder militärische Komponenten, auch wenn es sich dabei nur um individuelle Sekundierungen handelte und nicht um die Entsendung ganzer militärischer Einheiten. Militärbeobachter wurden etwa nach Moldau (seit 1993) und Georgien (2008) entsendet. Zivile Grenzbeobachter wurden entlang der georgisch-russischen Grenze (1999-2005) eingesetzt, und zivile Polizeiberater waren Teil der Community Security Initiative in Kirgisistan (2010-2015).

Für Friedensförderung wurden nationale Polizeikräfte durch die OSZE-Mission in Kroatien sowie im Rahmen der Community Security Initiative in Kirgisistan ausgebildet und gestärkt. Die Mission in Skopje mit ihrem Public Safety and Community Outreach Department widmet sich nach wie vor den Themen Polizeiausbildung sowie Kontrolle und Reformen der Polizei. Zudem unterstützt sie die Polizei beim Kampf gegen den illegalen Waffenhandel und die organisierte Kriminalität. ${ }^{4}$

Während sich die OSZE-Feldoperationen in Zentralasien und auf dem Westbalkan auf die Stärkung von Resilienz und guter Regierungsführung konzen- 
triert haben, dienten die Missionen in Bosnien und Herzegowina, im Kosovo und in Nordmazedonien dem Zweck, die Umsetzung der Friedensverträge und/ oder UN-Resolutionen mit dem Ziel der Konfliktverhütung und -lösung zu unterstützen.

\section{Die OSZE-Missionen zur Waffenstillstandsbeobachtung}

Bisher gab es nur zwei OSZE-Missionen, die mit Friedensoperationen militärischer Art vergleichbar wären: die Kosovo-Verifikationsmission (KVM) und die Sonderbeobachtermission in der Ukraine (SMM). Diese beiden zivilen Missionen zur Waffenstillstands-Beobachtung begannen jeweils unter eher ruhigen Umständen, endeten aber letztendlich in aktiven Konfliktsituationen, in denen es keinen Frieden mehr gab, der zu sichern gewesen wäre. Die Entsendung der KVM nach Jugoslawien fand während der sich zuspitzenden Kosovokrise im Jahr 1998 statt, als die USA und die NATO angesichts der Gewalttaten vor Ort damit drohten, militärisch gegen das MiloševićRegime vorzugehen. Das Mandat der KVM - das ohne die OSZE ausgehandelt worden war und aus dem HolbrookeMilošević-Abkommen abgeleitet wurde forderte beide Seiten dazu auf, die Gewalttaten zu beenden, ihre bewaffneten Streitkräfte aus dem Kosovo abzuziehen und einen Waffenstillstand einzuhalten. Die KVM sollte etwa zweimal so groß sein wie die gegenwärtige SMM, konnte ihre Präsenz aber nicht schnell genug anpassen, um den zunehmenden Sicher- heitsbedrohungen gewachsen zu sein. Im März 1999, sechs Monate nach ihrer ursprünglichen Entsendung, musste die Mission aus Sicherheitsgründen in die benachbarte Ehemalige Jugoslawische Republik Mazedonien (EJRM - heute Nordmazedonien) verlegt werden, bevor sie schließlich beendet wurde.

Die SMM wurde Anfang 2014 als ein präventiver Einsatz entwickelt, der auf Deeskalation, Vertrauensbildung und Dialogförderung in der gesamten Ukraine ausgerichtet war. Innerhalb weniger Wochen fand sich die Mission jedoch in einem bewaffneten Konflikt wieder und musste sich an neue Sicherheitsbedingungen und neue Aufgaben zur Beobachtung des Waffenstillstands auf Grundlage der Minsker Vereinbarungen anpassen. Seitdem ist die SMM zu einem wichtigen Instrument der Krisenbewältigung in der Ukraine geworden.

Im Folgenden werde ich auf sechs wesentliche Eigenschaften dieser beiden Missionen eingehen, wobei ich dabei den Schwerpunkt auf die SMM legen werde. Das Ziel ist es, Erkenntnisse und bewährte Praktiken zu identifizieren sowie Empfehlungen für zukünftige OSZE-Missionen und andere regionale oder internationale Missionen unter VN-Mandat zu formulieren.

\section{Missionsmandate}

Das Mandat der KVM wurde ohne Beteiligung der OSZE verhandelt. Dies führte zu einer Diskrepanz zwischen dem politischen Prozess, der operativen Planung und der strategischen Kontrolle. 
Die Agenda der Mission war außerordentlich breit angelegt. Sie umfasste das Beobachten des Waffenstillstandes, die Untersuchung entsprechender Verstöße, die Unterstützung der Rückkehr von Vertriebenen sowie die Überwachung der Regierungsarbeit und der Wahlen. Diese Vielzahl an Aufgaben führte gemeinsam mit organisatorischen Defiziten da$\mathrm{zu}$, dass die KVM-Führung nicht in der Lage war, einen einheitlichen Plan zu deren Umsetzung zu entwickeln. ${ }^{5}$

Im Gegensatz dazu wurde das SMMMandat vom Schweizer OSZE-Vorsitz gemeinsam mit weiteren Teilnehmerstaaten im Zuge der Maidan-Krise 2014 entworfen und verhandelt. Trotz eines äußerst aufgeheizten politischen Klimas wurde das Mandat einstimmig verabschiedet und genoss bis zum Abschluss der vorliegenden Analyse große Unterstützung. ${ }^{6}$ $\mathrm{Da}$ das Mandat breit angelegt ist, konnte der geographische Geltungsbereich der Mission flexibel gehandhabt werden was angesichts der illegalen Annexion der Krim durch Russland unverzichtbar war. Dennoch weist das Mandat einige Schwächen auf. In bestimmten Bereichen - etwa was die Dialogförderung betriff hätte eine präzisere Sprachwahl zu mehr Synergien mit anderen laufenden OSZEInitiativen in der Ukraine führen können. Ein weiteres Defizit ist die fehlende Berücksichtigung einer Geschlechterperspektive innerhalb der Missionsaktivitäten.

Die Flexibilität des SMM-Mandats ermöglichte es, schnell auf Konfliktdynamiken vor Ort zu reagieren. Obwohl sich die Mission seit 2014 nicht verändert hat, wurden ihr zusätzliche Aufga- ben zugewiesen, etwa durch die Trilaterale Kontaktgruppe (TKG) oder das Normandie-Format. Der OSZE-Bericht „A Peaceful Presence“ verdeutlicht, dass die Kapazitäten der Organisation zur Planung von Missionen immer noch sehr begrenzt sind. Deswegen besteht die Gefahr, dass auf kurzfristig auftretende Notfälle mit bloßen Verlegenheitslösungen reagiert wird. Für langfristige Planungen und Strategien bestehen kaum Kapazitäten und Möglichkeiten. ${ }^{7}$

\section{Einsatzbedingungen}

Sowohl die KVM als auch die SMM befanden sich in der paradoxen Situation, im Rahmen einer zivilen Friedensmission mit militärischen Herausforderungen umgehen zu müssen. ${ }^{8}$ Während der kurzen Planungsphase für die KVM im Jahr 1998 waren die Führungskräfte der OSZE nicht in der Lage, klare Anweisungen zu geben, wie das Mandat umzusetzen sei. Außerdem ging der Rekrutierungsprozess (vor allem für die Sekundierung von Experten mit Erfahrung bei der Waffenstillstandsverifizierung und Polizeiarbeit) schleppend voran, trotz eines entsprechenden Aufrufs der UN an alle OSZETeilnehmerstaaten. ${ }^{9}$ Nach drei Monaten waren 700 Beobachter im Einsatz. Als die Mission im März 1999 aufgrund von Sicherheitsbedenken beendet wurde, war die Zahl der Beobachter auf 1400 gestiegen. Der KVM fehlte von Anfang an eine adäquate institutionelle Unterstützung, die für eine beschleunigte Aufstellung von Einsatzkräften und Beobachtern erforderlich gewesen wäre. Auch eine be- 
sondere Sorgfaltspflicht, die gerade für eine so ambitionierte und in einem aktiven Konfliktgebiet tätige Feldoperation notwendig gewesen wäre, war nicht vorhanden.

Im Gegensatz dazu konnte sich die SMM von Anfang an auf die Unterstützung durch das OSZE-Sekretariat und insbesondere auf das Konfliktverhütungszentrum (KVZ) verlassen. Diese Unterstützung ermöglichte es der Mission, ihre Operationen, ihr Personal und ihre Materialbeschaffung immer wieder anzupassen. Dank der im Rahmen der KVM gewonnenen Erfahrungen sowie der Befugnisse durch Ministerratsbeschluss Nr. 3/11 zum Konfliktzyklus ${ }^{10}$ konnte sich die SMM darauf verlassen, dass folgende Elemente bereits vorhanden waren:

- ein operativer Rahmen für Krisenreaktion und Personal,

- eine Liste verfügbarer Mitarbeiter, die schnell entsendet werden können,

- ein virtueller Ausrüstungspool. ${ }^{11}$

\section{Unparteiliche Berichte und Verhaltensweisen}

Unparteilichkeit ist eine entscheidende Voraussetzung für jede OSZE Feldmission - ebenso wie ein Konsens und ein gewaltfreies Vorgehen. Ende der 1990er Jahre war die OSZE in einen anhaltenden Machtkampf zwischen dem Milošević-Regime und der kompromisslosen US-Diplomatie verstrickt, die vom UN-Sicherheitsrat und der Nato unterstützt wurde. Der gemeinsame Beschluss, eine OSZE-
Mission zu entsenden, war Teil eines Abkommens, das auch eine Überwachung Jugoslawiens aus der Luft durch die NATO beinhaltete. Die Sicherheit der KVM hing von einer sogenannten NATO Extraction Force in der benachbarten EJRM ab. ${ }^{12}$ Aufgrund der Ernennung des US-Diplomaten William Walker als Missionschef war es der OSZE nicht mehr möglich, unparteiisch und neutral zu bleiben, da sich die USA eindeutig als Gegner des Milošević-Regimes positioniert hatten. Dies zeigte sich in dramatischer Weise, als der Missionschef im Gastgeberstaat zur Persona non grata erklärt wurde. ${ }^{13}$

Im Gegensatz dazu ist die SMM nicht aus „Brinkmanship“- Diplomatie hervorgegangen und konnte daher ihre Unparteilichkeit wahren. Obwohl sie gelegentlich kritisiert wurde, hat die SMM entgegen der üblichen UN-Praxis eine „Politik der Nicht-Zuweisung" betrieben, also ein „Berichten der Fakten, ohne dabei Schuldzuweisungen vorzunehmen, und Probleme im Bereich harte Sicherheit mit den humanitären, politischen und sozioökonomischen Konsequenzen des Konflikts abzuwägen “. ${ }^{14}$ Dennoch gab es hartnäckige Versuche, die Mission politisch in Bezug auf Sorgfaltspflichtfragen oder Schuldzuweisungen bei Verstößen gegen den Waffenstillstand zu beeinflussen auch von nationalen OSZE-Delegationen.

\section{Vor- und Nachteile der Nutzung moderner Technik}

Während die KVM eine „Low Tech“-Mission war, erwarb die SMM auf politi- 
schen Druck des amtierenden Vorsitzes hin moderne technische Ausrüstung wie etwa Kameras, Satellitenbildgeber, akustische Sensoren und Drohnen (UAV, unarmed aerial vehicles, unbemannte Luftfahrzeuge), um die von ihren Bodenpatrouillen gesammelten Informationen $\mathrm{zu}$ ergänzen. Die Nutzung solcher Technologien durch die SMM wurde durch das Minsker Waffenstillstandsabkommen ermöglicht. Der OSZE fehlte es aber an Wissen über die Anschaffung oder Nutzung von Drohnen. Sie traf deshalb eine Vereinbarung mit einem privaten Unternehmen, wodurch sämtliche technische Dienstleistungen und die notwendige Expertise dafür ausgelagert wurden, aber dennoch unter Kontrolle der SMM blieben. ${ }^{15}$

Der Einsatz von UAVs erleichterte die Arbeit der Mission, da sie auf diese Weise Aufgaben übernehmen konnte, die ansonsten von Patrouillen in gefährlichen Gebieten oder verbotenen Bereichen durchgeführt worden wären. Die Fernüberwachung spielte auch während der COVID-19-Pandemie eine wichtige Rolle, da sie Versuche erschwerte, die Pandemie als Vorwand für Lockdowns, Zugangsverweigerungen und Schließungen von „Grenzen“ zu nutzen.

Der Einsatz von Drohnen in aktiven Konfliktzonen ist gefährlich und teuer. Die Drohnen werden oft elektronisch gestört oder beschossen - was einen klaren Verstoß gegen die Minsker Vereinbarungen darstellt. Vom ersten Drohnenflug im Oktober 2014 bis zum 31. März 2019 verlor die SMM 39 UAVs. Davon gingen 67 Prozent in Gebieten verloren, die nicht von der Regierung kontrolliert wer- den, 31 Prozent in Gebieten, die von der Regierung kontrolliert werden, und zwei Prozent entlang der Kontaktlinie. ${ }^{16}$

Mit dem zunehmenden Einsatz moderner Technik sind auch Probleme im Zusammenhang mit der Verarbeitung und Nutzung der gesammelten Daten aufgetreten. Die SMM musste feststellen, dass sie zu schlecht ausgestattet war, um eine große Menge an Informationen aus verschiedenen Quellen zu verarbeiten. Zudem dauerte der Zugriff der Beobachter auf Satelliten- und Drohnenbilder oft zu lange, als dass er für die Planung von Patrouillen und die Umsetzung von Maßnahmen effektiv gewesen wäre. Ein neues Informations-Managementsystem musste für die Zusammenführung von Daten und die Vorbereitung von Entscheidungen, Plänen und öffentlichen Berichten eingeführt werden. ${ }^{17}$

\section{Rechtsstatus und Sorgfaltspflicht}

Der Umstand, dass die OSZE keine Rechtspersönlichkeit besitzt, ist ein schweres Hindernis für ihre Feldmissionen. Die SMM konnte ihre Feldpräsenz erst dann wie geplant aufbauen, als eine Absichtserklärung mit der Gastgeber-Regierung zustande gekommen war - mehrere Wochen nach Beginn der Mission. ${ }^{18}$ Noch problematischer war, dass die OSZE während dieser ersten Wochen ohne rechtlichen Schutz in der Ukraine für jeden verursachten Schaden zur Rechenschaft gezogen werden konnte. Während dieser Phase wurden acht Beobachter von einer Gruppe Kosaken als Geiseln genommen. Dabei stellte sich die Frage, 
wer dafür haften würde, falls es zu Personenschäden kommen sollte: der Missionschef, der Generalsekretär, der amtierende Vorsitz oder vielleicht die sekundierenden Teilnehmerstaaten? Bis heute sind solche Fragen nach der Sorgfaltsplicht nicht geklärt. ${ }^{19}$ Ein Problem der SMM ist auch, dass der Schutz ihres Personals von Garantien der Kriegsteilnehmer abhängt. Im Gegensatz zur KVM verfügt die SMM über keine eigene militärische Evakuierungseinheit. Eine komplexe Konfliktumgebung erfordert es, dass Vorkehrungen zur Sorgfaltspflicht Teil der Missionsplanung sind. Leider war dies bei beiden Friedensmissionen der OSZE nicht der Fall. Im Fall der KVM blieb zum Beispiel die Bitte der OSZE um eine mobile medizinische Betreuung - einschließlich Militärambulanz und gepanzerter Fahrzeuge - nach der Entsendung der Mission monatelang unbeantwortet. $^{20}$

\section{Partnerschaften für gemeinsame oder hybride Missionen}

Bisher gab es noch keine gemeinsamen Missionen der OSZE mit anderen internationalen Organisationen. Die Europäische Sicherheitscharta von Istanbul aus dem Jahr 1999 würde es der OSZE jedoch ermöglichen, mit anderen regionalen und internationalen Organisationen zusammenzuarbeiten, auch bei der Friedenssicherung. ${ }^{21}$ Im Jahr 2014 wurde ein Vorschlag für eine hybride oder „erweiterte" SMM gemacht, als Deutschland, Frankreich, Italien und die Russische Föderation jeweils anboten, der Mission
Drohnen und militärische Ausrüstung zur Verfügung zu stellen. Zu diesen Angeboten der einzelnen Länder gehörte auch ein Schutz durch militärische Einheiten. Die Einbeziehung nationaler militärischer Einheiten in die SMM hätte jedoch den zivilen und unparteilichen Charakter der Mission untergraben können. Zudem wären dafür ein zusätzlicher Beschluss des Ständigen Rats sowie ein bilaterales Stationierungsabkommen für Streitkräfte mit der Ukraine nötig gewesen. ${ }^{22}$

Ein weiterer Vorschlag für eine hybride Operation - ebenfalls in der Ukraine - kam 2017 aus Russland. In einem Entwurf für eine Resolution des UN-Sicherheitsrats schlug Russland vor, die SMM entlang der Kontaktlinie durch eine leicht bewaffnete UN-Friedenstruppe zu schützen..$^{23}$ Die Ukraine lehnte die Möglichkeit einer hybriden UN-OSZEMission ab und schlug im Gegenzug bei der Generalversammlung der Vereinten Nationen 2017 eine robuste friedenserzwingende Mission gemäß Kapitel VII der Charta der Vereinten Nationen vor. Dadurch wäre die SMM überflüssig geworden. ${ }^{24}$ Beide Vorschläge fanden wenig Zuspruch und wurden aufgegeben.

\section{Wie auf die Erfolgsgeschichte der SMM aufgebaut werden kann}

Die SMM hat bewiesen, dass sie seit ihrer Entsendung 2014 in der Lage ist, viel zu lernen. Trotz ständiger Verstöße gegen den Waffenstillstand, Zugangsverweigerungen und der COVID-19-Pandemie hat sie ihre Arbeit auf beiden Seiten 
der Kontaktlinie fortgesetzt - wenn auch teilweise stark eingeschränkt. Im Laufe der Jahre haben sich Forscher und Fachleute mit der Mission beschäftigt, deren Effektivität untersucht und größtenteils gelobt. $^{25}$

Angesichts des ermutigenden Beispiels der SMM ist es denkbar, dass die Teilnehmerstaaten auch einer ähnlichen Mission in einem anderen Land zustimmen würden, sollte eine veränderte Sicherheitslage und politische Situation dies erfordern. Die naheliegendste Möglichkeit wäre eine Operation in der Region Bergkarabach. Sicher: Die Ko-Vorsitzenden der Minsk-Gruppe und die HLPG wurden durch die Entsendung der russischen Friedenstruppe an den Rand gedrängt. Eine Expertengruppe argumentiert dennoch, dass eine multinationale OSZE-Friedenstruppe immer noch benötigt wird, „denn es könnte sein, dass Moskau im Laufe der Zeit die Kosten und Belastungen nicht mehr tragen will, die es sich nun allein aufgeladen hat". ${ }^{26}$ Eine mögliche Beilegung des Bergkarabach-Konflikts könnte also eine zivile OSZE-Friedensmission erfordern, um die Einhaltung von Verpflichtungen zu beobachten und Aktivitäten zum Friedensaufbau zu unterstützen. Dies wäre von den politischen Entwicklungen abhängig und könnte Teil eines breitangelegten Rahmenwerks sein.

\section{Schlussfolgerungen und Empfehlungen}

Im Vergleich zu den klassischen militärischen UN-Friedensmissionen haben zivile Operationen einen leichten „Foot- print", was ein Vorteil ist. Zivile Missionen setzen außerdem keine Zwangsmittel ein, sondern agieren als Vermittler. Dadurch ist es leichter, einen Konsens für ihr Mandat zu erreichen, vom Gastgeber-Staat akzeptiert zu werden und unparteiisch zu bleiben. Entscheidend ist hier, eine effektive Abstimmung zwischen politischer und operativer Ebene bei der Mandatswahrnehmung zu garantieren und gleichzeitig die Möglichkeit zu haben, die Mission besser zu schützen, falls sie in einer bewaffneten Konfliktzone operiert. ${ }^{27}$

Das Mandat und die zusätzlichen Aufgaben der SMM gehen deutlich über das Beobachten von Waffenstillständen hinaus und beinhalten das Beobachten und Berichten von Verstößen gegen die Menschenrechte und die ökologische Sicherheit. Gleichzeitig bietet sie Good Offices und Vermittlungsdienste an. ${ }^{28}$ Der Einsatz der OSZE für diesen ganzheitlichen Ansatz hat sich als Vorteil erwiesen. Heutige Friedensmissionen müssen sich zunehmend mit Themen wie Instabilität, fragile Staaten und Governance in allen Phasen des Konfliktzyklus beschäftigen.

Die aktuellen geopolitischen Trends lassen vermuten, dass Friedensmissionen als Instrumente für die Konfliktverhütung und das Krisenmanagement auch in den kommenden Jahren gefragt sein werden. Bei der Planung einer solchen Mission (oder auch einer anderen OSZE-Mission) sollten folgende Empfehlungen berücksichtigt werden, die auf den bei früheren Missionen (vor allem der SMM) gewonnenen Erfahrungen und bewährten Praktiken beruhen: 
- Ausarbeitung eines flexiblen Missionsmandates: Missionsmandate sollten nur in den Bereichen Vorschriften machen, in denen dies nötig ist. Ein gutes Missionsmandat erleichtert der Missionsleitung eine ordentliche Planung und Strategieentwicklung. Ein Kompromiss beziehungsweise ein Konsens für das Mandat kann auch durch interpretative Erklärungen erreicht werden, so wie dies bei der SMM der Fall war. Eine explizite Erwähnung von Geschlechterparität und Geschlechterperspektiven sollte ein wesentlicher Bestandteil des Mandats sein.

- Sicherstellung einer zuverlässigen institutionellen Unterstützung für die Entsendung der Mission: Die Wichtigkeit einer soliden institutionellen Unterstützung wurde durch die Unfähigkeit der KVM, rasch Experten mit der passenden Qualifikation zu entsenden, verdeutlicht. Eine schnelle Sekundierung von zivilem Personal mit geeigneten militärischen Kenntnissen hängt jedoch mehr von den Teilnehmerstaaten $a b$ als von der OSZE selbst. Listen für rasche Entsendungen und neue interne Standardvorgehensweisen (SOPs, Standard Operating Procedures) für das gesamte Sekretariat sind hilfreich für eine rasche Reaktion..$^{29}$ Ein verstärktes Planungsteam im Sekretariat könnte effizientere Vorsorgemaßnahmen treffen und die Planung der Mission besser umsetzen - dies gilt nicht nur für den Fall einer möglichen Mission in Bergkarabach, sondern auch für andere Missionen. Es wäre überlegenswert, ob die HLPG enger an die Arbeit des KVZ angebunden werden könnte, und damit höhere Planungskapazitäten innerhalb des Sekretariats und quer durch das gesamte Missionsspektrum erreicht werden könnten.

- Wahrung der Unparteilichkeit: Die Nationalität des Missionsleiters spielt in diesem Kontext durchaus eine Rolle. Zusätzlich sollten Missionsberichte eine SOP beinhalten, die dazu auffordert, „keine Schuldzuweisungen“ vorzunehmen. Die OSZE sollte Verfahren für den Umgang mit nicht-anerkannten Akteuren auf Grundlage des OSZE-Dokuments „Stabilisierende Maßnahmen für örtlich begrenzte Krisensituationen" 30 entwickeln.

- Nutzung moderner Technologien: Die Beobachtung mit technischen Mitteln ist $\mathrm{zu}$ einem besonderen Merkmal der SMM geworden. Durch das Beobachten aus der Ferne können gefährliches Terrain und unsichere Checkpoints vermieden werden. In Kombination mit einer verbesserten Datenverarbeitung und operativen Planung können Missionen dadurch effektiver und sicherer gemacht werden. Plattformen zur Datenverarbeitung und die entsprechenden Fachkenntnisse sollten von Anfang an zur Verfügung stehen. Die OSZE-Feldmissionen können von den Erfahrungen der SMM profitieren und technologische Mittel auch für andere Aufgaben nutzen, wie etwa für die Risikobeurteilung von Naturkatastrophen.

- Kohärenz von Sorgfaltspflicht und Missionsplanung: Für jede zivile Feldmission, die in einer Hochrisiko- 
Umgebung tätig ist, sollten von Anfang an eine Sorgfaltspflicht-Strategie und entsprechende Überwachungsmaßnahmen existieren.

- Berücksichtigung der Risiken gemeinsamer Missionen: Missionen, die gemeinsam mit anderen Organisationen ausgeführt werden, sind zwar denkbar, aber politisch schwer machbar. Vereinbarungen für gemeinsame Einsätze mit der Polizei oder Armeekontingenten würden die Planungskapazitäten der OSZE stark beanspruchen und ihre Unparteilichkeit potenziell gefährden. Zudem wäre dafür eine Resolution des UN-Sicherheitsrats erforderlich.

- Wahrung einer engen Verbindung zwischen den politischen und operativen Prozessen: Das Schicksal der KVM hat gezeigt, wie wichtig es ist, dass die Leiter der Feldoperationen am politischen Prozess beteiligt sind. Damit wird eine Aufsplitterung der Verantwortlichkeiten vermieden - eine ständige Gefahr bei komplexen Operationen. Im Fall der SMM wurde dieses Problem gelöst, indem der Leitende Beobachter der SMM aktiv in den Minsk-Prozess einbezogen wurde (als Koordinator für die Arbeitsgruppe Sicherheit der Trilateralen Kontaktgruppe).

- Mehr Bewusstsein schaffen für die Vorzüge der OSZE und ihrer Operationen: Angesichts ihres umfangreichen Erfahrungsschatzes sollte die OSZE mehr in ihren institutionellen Lernprozess und ihre OutreachAktivitäten investieren. Es ist wichtig, dass die Teilnehmerstaaten und andere internationale Organisationen mehr Vertrauen in die Fähigkeit der OSZE entwickeln, Krisen zu bewältigen und Friedensmissionen zu betreiben - auch in Zonen mit kriegerischen Auseinandersetzungen.

\section{Endnotes}

„Friedensoperationen“ soll hier als Oberbegriff für Friedenssicherung, Friedensmissionen, politische Sondermissionen und Feldoperationen dienen. In der entsprechenden Literatur werden „Friedenssicherung“ und „Friedensmissionen“ oft gleichbedeutend verwendet.

„Multilateral peace operations“, Stockholm International Peace Research Institute, 2020, https://www.sipri.org/sites/def ault/files/2020-06/mpo20_fill.pdf

3 Das Kapitel III des Helsinki-Dokuments (1992) enthält nach wie vor die wichtigsten Rahmenbedingungen für OSZE-Aktivitäten im Bereich der Friedenssicherung. Siehe KSZE Helsinki-Dokument 1992: Herausforderung des Wandels, 10. Juli 1992, https://www.osce.org/files/f /documents/c/1/39532.pdf

Für weitere Informationen über OSZE-Feldoperationen siehe: OSCE, Survey of OSCE Field Operations, SEC.GAL/111/20/Corr.1*, 26 August 2020, https://www.osce.org/secretariat/ 74783

Alex J. Bellamy/Stuart Griffin, „OSCE Peacekeeping: Lessons from the Kosovo Verification Mission", in: European Security $1 / 2002,1-26$, S. 22.

$6 \mathrm{Zu}$ Beginn wurde die Mission alle sechs Monate verlängert. Seit März 2015 wird das Mandat der SMM für einen Zeitraum von je 12 Monaten verlängert.

7 OSCE, A peaceful presence - the first five years of the OSCE Special Monitoring 
Mission to Ukraine, 29 June 2021, S. 53, https://www.osce.org/secretariat/491220

Dazu gehörten die Beobachtung von Waffenstillständen; die Untersuchung von Verstößen gegen den Waffenstillstand; die Ermöglichung, Verifikation und Beobachtung des Rückzugs schwerer Waffen; das Aushandeln örtlicher Waffenstillstände an der Front (so genannte „Fenster des Schweigens“) sowie der Kontakt zu Koordinierungsstellen zwischen militärischen Einheiten.

Paragraph 7 der Resolution 1203 des UNSicherheitsrats (1998) „fordert die Staaten und die internationalen Organisationen nachdrücklich auf, der OSZE-Verifikationsmission im Kosovo Personal zur Verfügung zu stellen"; siehe UNSC, Resolution 1203 (1998), S/RES/1203 (1998), 24. Oktober 1998, https://digitallibrary.u n.org/record/262334? In=en

10 Beschluss Nr. 3/11 des OSZE-Ministerrats über Elemente des Konfliktzyklus, 7. Dezember 2011, https://www.osce.org/fil es/f/documents/9/d/88841.pdf

11 Michael Raith, Addressing the Conflict Cycle: The OSCE's Evolving Toolbox, IFSH (ed.), OSCE Insights 3 (Baden-Baden: Nomos, 2020), https://doi.org/10.5771/97 83748922339-03, S. 48.

12 NATO Press Statement, (98)139, 5 December 1998, https:/www.nato.int/doc u/pr/1998/p98-139e.htm

13 OSCE Chairmanship, „Head of Kosovo Verification Mission declared 'persona non grata”, OSCE, 19 January 1999, https://www.osce.org/cio/52376

14 OSCE, a.a.O. (Fußnote 7), S. 67.

15 Dies beinhaltete ein Vereinbarungspaket mit einer privaten Firma, das zu Beginn zwei Bodenkontrollstationen, vier Langstreckendrohnen, sechs Bediener und drei für die Wartung zuständige Mitarbeiter umfasste. Siehe OSCE, a.a.O. (Fußnote 7), S. 49.

16 OSCE, a.a.O. (Fußnote 7), S. 50.
17 Die Umsetzung des sogenannten „Enterprise Geographic Information System“ ist bis zum Druck dieses Artikels nicht abgeschlossen.

18 Keinen juristischen Status innezuhaben hat den Beschaffungsprozess und die Entsendung der Mission in die Ukraine erschwert. Zudem verhinderte dieser Umstand, dass die Mission Bankkonten eröffnen, Verträge abschließen und wichtige Ausrüstungsbestandteile importieren konnte. Siehe OSCE, a.a.O. (Fußnote 7), S. 20.

19 Seitdem die SMM-Beobachter zum ersten Mal physisch bedroht wurden, hat sich in Bezug auf die Sorgfaltspflicht bei OSZE-Missionen viel getan; siehe Maarten Merkelbach, Voluntary guideline on the duty of care to seconded civilian personnel, Swiss Federal Department of Foreign Affairs, UK Stabilisation Unit and the German Center for International Peace Operations, 2017, https:/www.eda. admin.ch/dam/eda/en/documents/aussen politik/menschenrechte-menschliche-sich erheit/Voluntary_Guidelines_on_the_Du ty_of_Care_to_Seconded_Civilian_Perso nnel_Final_170420.pdf

20 Larissa Daria Meier, A role for OSCE peacekeeping? From the 1992 Helsinki Guidelines to the Special Monitoring Mission to Ukraine, CORE/IFSH Working Paper 27, 2015, S. 33, https://ifsh.de/fi le-CORE/documents/Working_Papers/C ORE_WP27.pdf

21 OSZE, Dokument von Istanbul 1999 (Europäische Sicherheitscharta und Erklärung des Ministerrats von Istanbul), https://www.osce.org/mc/39569

22 Deutschland z.B. bot an, ein Kontingent mit einer LUNA-Aufklärungsdrohne zu entsenden. Das Kontingent hätte - inklusive logistischer, medizinischer und anderer Elemente - mehr als 200 bewaffnete Soldaten umfasst.

23 Siehe International Crisis Group, Can peacekeepers break the deadlock in 
Ukraine?, Europe Report N²46, 15. Dezember 2017, S. 2, https://d2071andvip0 wj.cloudfront.net/246-can-peacekeepers-b reak-the-deadlock-in-ukraine.pdf

24 Fred Tanner, Sustaining civilian missions in conflict zones: The case of the OSCE Special Monitoring Mission to Ukraine, swisspeace, Policy Brief 03, 2018, S. 2, https://www.swisspeace.ch/assets/publica tions/downloads/Policy-Briefs/6656f9004 2/Sustaining-Civilian-Missions-in-Conflic $\mathrm{t}$-Zones-Policy-Brief-18-swisspeace-fred_t anner.pdf

25 Siehe z.B. Walter Kemp, „Civilians in a War Zone: The OSCE in Eastern Ukraine", in: IFSH (ed.), OSCE Yearbook 2017, Baden-Baden: Nomos, 2018, 113-123; André Härtel/Anton Pisarenko/ Andreas Umland, „The OSCE's Special Monitoring Mission to Ukraine: The SMM's Work in the Donbas and Its Ukrainian Critique in 2014-2019”, in: Security and Human Rights June/2021, 1-34; OSCE, a.a.O. (Fußnote 7).
26 Global Europe Program Working Group on the Future of the OSCE, Uncommon cause: The future of the OSCE, The Wilson Center, February 2021, S. 7, https://w ww.wilsoncenter.org/sites/default/files/m edia/uploads/documents/Uncommon Cause - The Future of the OSCE v2.pdf

$27 \mathrm{Zu}$ Schutz gehören eine Priorisierung militärischer Fähigkeiten bei der Rekrutierung, ein fachspezifisches Training, Kenntnisse in der medizinischen Evakuierung, die Bereitstellung von gepanzerten Fahrzeugen mit GPS, kugelsicheren Westen und Helmen und eine Satellitenkommunikation. Siehe Daria Meier, a.a.O. (Fußnote 20), S. 46.

28 OSCE, a.a.O. (Fußnote 7), S. 17.

29 Raith, a.a.O. (Fußnote 11), S. 48.

30 OSZE, Stabilisierende Maßnahmen für örtlich begrenzte Konfliktsituationen, DOC.FSC/2/96, 25. November 1993, https://www.osce.org/fsc/41316 